\title{
EFECTO DEL AMBIENTE DIGITAL SIMAS EN LA COMUNICACIÓN, LA ACTITUD Y LAS ESTRATEGIAS PEDAGÓGICAS UTILIZADAS POR DOCENTES Validación de dos áreas y niveles del sistema educativo
}

Effect of the SIMAS Digital Environment in the Communication, Attitude and the Pedagogical Strategies Used by Teachers: Validation of two Areas and Levels of the Education System

\author{
Paola lucumi Useda ${ }^{1}$, Martha Alexandra González Castañeda ${ }^{1}$ \\ ${ }^{1}$ Escuela Normal Superior de Ubaté, Colombia
}

KEY WORDS

Teacher Communication Pedagogical Practices Teaching Strategies Teachers' Attitudes Information and Communication Technologies (ICT)
ABSTRACT

The article shows the project "effect of digital ambient SIMAS in the communication, the attitude and the pedagogics strategies used by teachers: validation of two areas and levels of educative system" It has been developed with experimental groups (used SIMAS) and control groups (using plane text in the Escuela Normal Superior de Ubate (ENSU) and the Fundacion de Educacion Superior Nueva America (FESNA) The advances are: 1. Definition of categories of strategies of study of teachers communication, teachers actitudes in learning spaces and practices of pedagogics strategies used by teachers. 2. Make and application of instruments for collected datas.

\section{PALABRAS CLAVE}

Comunicación docente

Prácticas pedagógicas

Estrategias pedagógicas

Actitud docente

Tecnología de la información y la comunicación (TIC)

\section{RESUMEN}

El proyecto "Efecto del ambiente digital SIMAS en la comunicación, la actitud y las estrategias pedagógicas utilizadas por docentes: validación de dos áreas y niveles del sistema educativo", Se desarrolla con grupos experimentales (usan SIMAS) y grupos control (usan texto plano), en la Escuela Normal Superior Ubaté (ENSU) y en la Fundación de Educación Superior Nueva América (FESNA). Los avances del proyecto son: 1) definición de categorías de estudio: Estrategias de comunicación docente; Actitud de docentes en ambientes de aprendizaje; y, Prácticas y estrategias pedagógicas utilizadas por los docentes, 2) Elaboración y aplicación de instrumentos para la recolección de datos.

\section{GLOBAL 我 KNOWLEDEGE}




\section{Presentación}

$\mathrm{E}$ l proyecto titulado: "Efecto del ambiente digital SIMAS (Sistema de Marcos para el Aprendizaje Significativo) en la comunicación, la actitud y las estrategias pedagógicas utilizadas por docentes: validación de dos áreas y niveles del sistema educativo", nace del Programa "Representación ontológica hipermedial en línea para el aprendizaje significativo", producto de la Convocatoria Nacional para la conformación de un banco de elegibles de programas de Ciencia, Tecnología e Innovación -CTeI- en innovación educativa con uso de las tecnologías de la información y la comunicación de Colciencias, identificado con el código 580-578-36197, cofinanciado por Colciencias; Ministerio de Educación Nacional de Colombia, MEN; Corporación Internacional de Redes de Conocimiento, ICONK; Escuela Normal Superior de Ubaté, ENSU; Fundación de Educación Superior Nueva América, FESNA; e, I3net. Su ejecución inició: el 30 de diciembre de 2013 y la fecha prevista de terminación es 30 de abril 2016. El trabajo se está llevando a cabo en Bogotá D.C. y Ubaté (Cundinamarca).

Actualmente las Tecnologías de la Información y la Comunicación, TIC, cada vez toman más relevancia para la formación tanto de docentes como de estudiantes, ya que facilitan y globalizan el conocimiento, lo cual repercute positivamente en la significatividad y calidad del proceso de educabilidad $y$ enseñabilidad, aportando entre otros muchos beneficios, la flexibilización de la enseñanza, el aprendizaje colaborativo y la capacidad de autoaprendizaje.

Teniendo como premisa que la aplicación de nuevas tecnologías en el contexto del aula de clase demuestra la ruptura de paradigmas que deberían generar cambios en la actitud, comunicación, prácticas y estrategias pedagógicas de docentes en ambientes de aprendizaje mediados por la tecnología, se evidencia la necesidad de crear nuevos escenarios que permitan realizar procesos de investigación al interior de las instituciones de educación sobre la creación de contenidos en el uso de TIC para la enseñanza al interior de las aulas de clase, mediante la aplicación de la plataforma SIMAS, como referente ejemplificador. Lo que se busca es identificar la aplicabilidad de este nuevo aplicativo en la metodología que utilizan los docentes durante el desarrollo de su ejercicio docente.

Las anteriores condiciones se están estudiando con docentes que se desempeñan en las áreas de Lenguaje, Desarrollo Humano, Práctica Pedagógica y en los niveles de educación preescolar, grado cero; básica, grado séptimo; media, grado undécimo; y, superior con el PFC, tercer semestre de la ENSU y en nivel de educación superior con el Programa de
Mercadeo de la FESNA, con el fin de validar no sólo la propuesta de SIMAS dentro de la metodología de clase en un entorno académico, sino además, contribuir a nuevos medios y mecanismos de difusión del conocimiento a través de aplicaciones tecnológicas que sean de fácil de acceso, entretenidas y, sobre todo, que permitan acceder al conocimiento de forma ágil, práctica y accesible para todos.

Se considera que es necesario apoyar procesos educativos con propuestas innovadoras, que contribuyan a que el docente optimice sus prácticas educativas cotidianas con el uso de TIC, ya que se evidencia en diferentes escenarios que algunos docentes continúan utilizando material tradicional que muchas veces, ya no permite la trasmisión, construcción o reconstrucción del conocimiento y tan solo se queda en la difusión de cierta información. Esto dificulta la comprensión y apropiación de temáticas y, por ende, el desarrollo de competencias; además, dificulta la aplicación de conceptos dentro de ambientes reales de trabajo colaborativo con pares e indica desarticulación en el proceso de aprendizaje.

La investigación está dirigida a la comprensión de procesos de comunicación, actitud y estrategias pedagógicas utilizadas por los docentes en el ambiente digital SIMAS, basado en la construcción de representaciones ontológicas, hipermediales y colaborativas y busca responder la pregunta: ¿Qué efecto genera una plataforma digital basada en la construcción de representaciones ontológicas, hipermediales y colaborativas en la escogencia de estrategias pedagógicas utilizadas por los docentes en procesos educativos específicos?

Para abordar la investigación y buscar respuesta al interrogante, se plantean tres fases metodológicas:

\section{Fase uno: preparación previa}

La elección y caracterización del grupo docente de investigación, en la que se definieron grupos control, mediante la utilización del texto plano y grupos experimentales que usan SIMAS, en los grados cero, séptimo, undécimo y tercer semestre de la formación profesional de la ENSU y II semestre del Programa de Mercadeo de la FESNA.

En esta primera fase se revisó el estado actual de la investigación a través de la elaboración del Estado del Arte, el cual se contempló desde tres categorías:

*Prácticas y estrategias pedagógicas que son utilizadas por los docentes, mediadas por TIC, definidas por Mockus (1984) como las acciones realizadas por el maestro con el fin de posibilitar la formación y el aprendizaje de las diferentes áreas del conocimiento adelantadas por los estudiantes.

*Actitud de docentes en ambientes de aprendizaje mediados por tecnología, apoyado este concepto en Curtis (1962) quien afirma que, las 
actitudes se encuentran siempre en relación con la predisposición de sentir, percibir, pensar y comportarse ante un objeto.

*Estrategias de comunicación docente en ambientes de aprendizaje; según Fonseca (2000) es una cualidad racional y emocional de los seres humanos que surge de la necesidad de ponerse en contacto con los demás, intercambiar información, transmitir ideas o intercambiar mensajes y/o significados que adquieren sentido de acuerdo a intereses y experiencias previas compartidas, mediante un conjunto común de símbolos.

Desde estas categorías teóricas, se logró determinar el punto de inicio de la estructuración metodológica de la investigación y la propuesta de la consecución de la información y la aplicación de instrumentos de medición. Para tal efecto, se hizo necesario realizar una actualización en procesos de investigación que el mismo Programa ofrece a sus colaboradores dentro del diseño del trabajo colaborativo que propone SIMAS.

Con el fin de fortalecer al equipo de la investigación, se realizó un diplomado de cualificación docente, propuesto por ICONK, en el uso del ambiente digital SIMAS y en el diseño de metodologías de investigación, en el que se contemplaron diversos temas relacionados con el proceso y escritura de trabajos científicos. En síntesis, las actividades propuestas para el diplomado abordan el campo de la redacción científica y la construcción de ontologías. Este diplomado a futuro será ofertado por las instituciones educativas participantes en la investigación, ENSU y FESNA.

\section{Fase dos: trabajo de campo}

En este momento de la investigación, se está midiendo el impacto en el desarrollo de la clase y, lo más importante, la forma como los estudiantes reciben el mensaje, la información y construyen el concepto mediante la utilización de la plataforma de SIMAS, en la presentación de por lo menos dos contenidos diferentes, con el objeto de determinar la viabilidad de la herramienta dentro del desarrollo académico de los estudiantes.

Con el ejercicio de observación de estrategias de comunicación de la aplicación al interior del aula y su articulación con la clase, se determinó que, en muchos casos, hay reticencia de algunos maestros, en cuanto al cambio de nuevas metodologías de enseñanza y simplemente replican el ejercicio de las ontologías y su aplicación en la plataforma SIMAS. Mientras que en otros casos, encuentran muy viable el ejercicio y la propuesta, aunque se les dificulta articularla con las temáticas y optan por recurrir al diseño de ejemplos y dinámicas muy diferentes a los conceptos que se deberían estar desarrollando al interior de la clase.
Este ejercicio se viene realizando con los docentes que laboran en las instituciones antes mencionadas, con el objeto de identificar los mecanismos de apropiación que tiene el docente frente al manejo y aplicación de la plataforma al interior del aula de clase. Esto demuestra la relevancia que tiene hacer el seguimiento de estas actividades para evaluar el desempeño tanto de los docentes como de SIMAS y demostrar su viabilidad para la enseñanza de diferentes conceptos.

\section{Fase tres: análisis e interpretación de resultados}

En esta etapa de la investigación se realiza el análisis e interpretación de la información desde el paradigma cualitativo y con base en los datos recolectados en la fase anterior. Dichos datos se organizan y sistematizan en matrices y se confrontan con las categorías teóricas desarrolladas en el estado del arte, buscando con ellos dar respuesta a la pregunta rectora del proyecto.

Siguiendo a Londoño (2014) para la investigación cualitativa la interpretación es un proceso connatural a ella, que consiste en conectar los datos que ya han sido analizados con la teoría apropiada, se hace necesario abstraer los datos relevantes, conformar una red de conceptos que logren sintetizar la naturaleza del fenómeno y establecer una asociación del fenómeno con otros fenómenos que sean similares o análogos.

El análisis que asume este proyecto a partir de la información recogida a través de los instrumentos de recolección de datos (entrevista a los maestros, fichas de observación de las clases, formatos de planeación de las clases utilizando el software SIMAS, encuestas a los estudiantes y pruebas de aptitud a los maestros) tiene un enfoque hermenéutico y se ha considerado que el modelo de análisis e interpretación más apropiado es la Teoría Fundamentada propuesto por Strauss y Corbin (2002) debido a que facilita acercarse a los niveles de descripción y permite confirmar las categorías teorías a través de la síntesis de hallazgos, estructuras, conceptos y categorías, compiladas en categorías emergentes desde la descripción endógena.

\section{Resultados Esperados}

Como resultados se espera: en primer lugar, contribuir a la formación de una comunidad virtual que se apropie de la plataforma SIMAS - Aula Inteligente para la construcción colaborativa del aprendizaje significativo, con proyección a la educación preescolar, básica, media, superior y universitaria.

En segundo lugar, crear herramientas de formación entre los docentes y estudiantes en 
formación de investigadores en la ENSU y en la FESNA, sobre el uso de ambientes digitales y colaborativos en línea que proyecten sus resultados a la educación básica, media vocacional y superior, con el fin de mantener una dinámica de evaluación de procesos de innovación de herramientas de educación, como el efecto que tiene el ambiente digital SIMAS, basados en la construcción de representaciones ontológicas, hipermediales y colaborativas en la comunicación, actitud y estrategias pedagógicas utilizadas por los docentes, con respecto a los desempeños y competencias que se definen en un proceso educativo específico.

En tercer lugar, durante el desarrollo de proyecto y una vez sea culminado, se busca contribuir a mejorar las prácticas pedagógicas, con el uso de la plataforma SIMAS, generando un impacto en la comunidad educativa con proyección a ser extensivo a nivel regional, considerando que es donde se pueden aplicar nuevas herramientas de enseñanza aprendizaje, mediante el desarrollo del uso didáctico de las TIC en los procesos pedagógicos $\mathrm{y}$, en tal sentido, crear redes de conocimiento para consolidar comunidades de aprendizaje y lograr que los estudiantes y los docentes puedan completar su desarrollo cognoscitivo a través de nuevas formas de enseñanza.

\section{Conclusiones}

Las conclusiones parciales que se pueden ir planteando en el desarrollo de la propuesta, a la fecha, son:

La actitud del maestro, la comunicación y las estrategias pedagógicas son componentes fundamentales en todos los procesos de formación y en ejercicio de maestros, pues generan un impacto fundamental $y$ definitivo en los procesos de enseñanza aprendizaje de los estudiantes.

El uso de nuevas tecnologías facilita y posibilita mayores y mejores niveles de comunicación, de más rápido acceso a la información; así mismo, puede y debe utilizarse en la escuela en pro del mejoramiento de la calidad educativa. De esta forma, es fundamental el papel de los directivos y docentes de las instituciones educativas, quienes son los encargados de gestionar y aprovechar los recursos y propiciar los medios favorables para los procesos formativos.

La tecnología facilita crear redes de conocimiento, pero es necesario que se haga de forma organizada y segura, para garantizar su sostenibilidad, ya que se crean muchas redes, pero a lo largo del tiempo son pocas las que se mantienen activas y realmente cumplen con el objetivo para el que fueron creadas.

El uso del software SIMAS, genera mayores niveles de compromiso en los maestros que lo implementan, logrando cambiar los paradigmas tradicionales en cuanto al manejo de los tiempos y los espacios de clase. Las ontologías impactan desde su presentación, permitiendo acceder a diversas y valiosas fuentes de información que aportan a la creación, construcción, reconstrucción y deconstrucción de conocimientos.

\section{Agradecimientos}

A los cofinanciadores del proyecto: Colciencias; Ministerio de Educación Nacional de Colombia, MEN; Corporación Internacional de Redes de Conocimiento, ICONK; Escuela Normal Superior de Ubaté, ENSU; Fundación de Educación Superior Nueva América, FESNA; e, I3net y Secretaría de educación de Cundinamarca.

A las compañeras y compañeros investigadores que con su experiencia, participación y acciones, hacen posible este proyecto. En especial a nuestras incondicionales compañeras: Sonia Yamile Suárez, María Liliana González, Nancy Esperanza Mateus, Inés Guacaneme Quiroga y sus grupos de estudiantes; a los docentes de la FESNA, Catalina Inés González y Juan Carlos Camacho, por compartir la experiencia de maravillarse con el conocimiento a través de SIMAS; a los docentes Ana Dolores Pachón y Alexis Páez docentes grupo control.

A la Dirección del Programa, Doctora Olga Lucía Londoño y Catalina Calderón.

Al Magister Juan José Cubillos, Rector de la Escuela Normal Superior de Ubaté, quien con ejemplo insta a los maestros a investigar. 


\section{Referencias}

Mockus, A. (1984). “Movimiento pedagógico y defensa de la calidad de la educación pública” En: Educación y Cultura. Bogotá, 2, 27-34.

Curtis, J.H. (1962). Psicología social. Barcelona: Grijalbo.

Fonseca, M. (2000). Comunicación Oral Fundamentos y Práctica Estratégica. México: Pearson Educación.

Londoño, O.L. (2014). Concepto y proceso de interpretación en investigación cualitativa. Material elaborado para el Diplomado en Virtualidad y Autonomía. Programa Representación ontológica hipermedial para el aprendizaje significativo. Bogotá.

Strauss, A. y Corbin, J. (2002). Bases de la investigación cualitativa: Técnicas y procedimientos para desarrollar la teoría fundamentada. Medellín: Universidad de Antioquia. 